our models will make other population genetics applications, such as inference of population history from patterns of $\mathrm{LD}$, both easier and more reliable.

The effect of gene conversion on LD also affects human genetics, a primary goal of which is to identify the genetic variants that affect susceptibility to complex diseases. Much recent work on the subject focuses on association mapping methods that use LD. Association mapping attempts to identify causal variants by typing many (e.g., thousands) single-nucleotide polymorphisms (SNPs) in a sample of unrelated individuals and then determining whether any of the SNPs are associated with the disease phenotype of interest. The rationale is that even if the SNPs that were typed do not directly affect disease susceptibility, they will be in strong LD with markers that do. The optimal marker density and success of association studies depend on the fine-scale structure of LD, and in particular on the expected decay of LD with physical distance. Jeffreys and May ${ }^{5}$ estimated that most recombination events are gene conversions $(\sim 80-94 \%$ of events) and that mean tract lengths (i.e., size of the converted piece) are small ( 55-290 bp). For these parameter values, gene conversion would more than double the effective recombination rate between closely spaced markers (e.g., ones within $2 \mathrm{~kb}$ of each other) but would have little effect on pairs of distant markers.

This might be important when trying to predict levels of LD between typed and untyped markers. Generally, when two nearby markers are in strong LD with each other, it is assumed that all markers in between are in strong LD with both end markers. This is the motivation for several recent definitions of 'haplotype blocks' (reviewed in ref. 7). But when the gene conversion rate is high and the marker density is low, it is possible that the intervening SNPs are not in strong LD with the end markers ${ }^{7}$ (Fig. 1). These SNPs can be thought of as 'holes' in haplotype blocks; these holes would reduce the efficacy of association studies. Further empirical and theoretical studies are needed to determine what practical effect, if any, this will have on future association studies.

\section{Recombination rate variation}

A more detailed assessment of the potential relevance of gene conversion to patterns of $\mathrm{LD}$ and to questions in population and human genetics would require data on gene conversion rates and conversion tract lengths from many other regions of the genome. Because estimating gene conversion parameters indirectly from patterns of LD is rather difficult to do accurately ${ }^{8}$, more direct experimental data, for example, from sperm typing, would be a welcome development.

Jeffreys and May ${ }^{5}$ chose to study known crossover hot spots in the MHC in part because the high recombination rates and high marker densities made direct parameter estimation much easier. But because the MHC hot spot regions may be atypical, it is not clear whether the gene conversion parameter estimates from these regions are applicable to the rest of the genome. The fraction of recombination events that are gene conversions may be higher in regions of low crossover rate, and mean conversion tract lengths may vary with levels of heterozygosity ${ }^{9-11}$. These empirical questions will eventually be answered. But comparable sperm typing studies in regions of the genome with average recombination rates (e.g., with crossover rates of $\sim 1.3 \mathrm{cM}$ per $\mathrm{Mb}$; ref. 12) will have to screen many more sperm to recover the same number of recombinants and, because of the lower marker densities, will be less informative about the distribution of conversion tract lengths.

For now, the results of Jeffreys and $\mathrm{May}^{5}$, together with recent work from the Jeffreys laboratory (and other laboratories), raise a host of other questions about recombination rate variation. What is the scale over which crossover and gene conversion rates vary across the human genome? How common are recombination hot spots? Are there regions that are hot spots for either crossover or gene conversion activity but not both? How strongly are male recombination rates, which can be estimated by sperm typing, correlated with female recombination rates? Given that there can be large differences in recombination rates across individuals ${ }^{2,13}$, there is also the possibility that there are large differences in recombination rates between different human populations.

1. Szostak, J.W. et al. Cel/ 33, 25-35 (1983).

2. Arnheim, N., Calabrese, P. \& Nordborg, M. Am. J. Hum. Genet. 73, 5-16 (2003).

3. Jeffreys, A.J. et al. Nat. Genet. 29, 217-222 (2001).

4. Zangenberg, G. et al. Nat. Genet. 10, 407-414 (1995).

5. Jeffreys, A. \& May, C. Nat. Genet. 36, 151-156 (2004).

6. Pritchard, J. \& Przeworski, M. Am. J. Hum. Genet. 69, 1-14 (2001).

7. Wall, J. \& Pritchard, J. Nat. Rev. Genet. 4, 587-597 (2003).

8. Ptak, S., Voelpel, K. \& Przeworski, M. Genetics (in the press).

9. Borts, R. \& Haber, J.E. Science 237, 1459-1465 (1987).

10. Langley, C. et al. Genetics 156, 1837-1852 (2000).

11. Andolfatto, P. \& Wall, J. Genetics 165, 1289-1305 (2003).

12. Kong, A. et al. Nat. Genet. 31, 241-247 (2002).

13. Jeffreys, A. \& Neumann, R. Nat. Genet. 31, 267-271 (2002)

\title{
Getting our AKT together in schizophrenia?
}

\author{
Joachim Hallmayer
}

\begin{abstract}
AKT1 is a protein kinase that functions as a central element in many pathways involved in the control of cell growth and apoptosis. Genetic and biochemical data now provide evidence that $A K T 1$ may be a susceptibility gene for schizophrenia.
\end{abstract}

Since Bleuler ${ }^{1}$ first introduced the term in 1911, schizophrenia has been the subject of controversy. The concept of schizophrenia

Joachim Hallmayer is in the Department of Psychiatry and Behavioral Sciences, Stanford University School of Medicine, Palo Alto, California 94304, USA. e-mail: joachimh@stanford.edu has changed substantially over time, and its boundaries in relation to other types of psychosis (bipolar disorder, for example) are far from clear. Discussion of the etiology of schizophrenia has shifted away from the mother-infant relationship to brain imaging and molecular genetics. But one finding has been replicated repeated: schizophrenia is familial, occurring at a higher frequency among relatives of individuals with the disorder. Coupled with the fact that the concordance rate is much higher in monozygotic twins than in dizygotic twins, this is compelling evidence of a genetic causation. With the advent of molecular approaches, identification of genes associated with schizophrenia has become the 'holy grail' in the field, and on page 131 of this issue, Effat Emamian and 
colleagues $^{2}$ identify $A K T 1$ as a susceptibility gene for schizophrenia.

\section{Genes and schizophrenia}

More than 20 genome-wide scans have been published and many more candidate genes investigated for a possible association with schizophrenia. What constitutes a 'real' positive association is a topic of heated debate.

There are nearly insurmountable difficulties in establishing convincing proof. First, there is the diagnostic problem. Diagnosis is based on a wide range of symptoms in such areas as inferential thinking, language, social behavior, volition and emotional expression. At one end of the spectrum are cognitively intact individuals describing a complex delusional system; at the other end are withdrawn, socially isolated individuals who are unable to express and take care of their own needs. Variability in diagnosis is inevitable. Second, the complex mode of inheritance suggests that there is no simple one-to-one relationship between genotype and phenotype. Third, schizophrenia is a disease of the brain. Unlike Alzheimer disease, no specific brain pathology has been described for schizophrenia, and the observed abnormalities are subtler and not restricted to a particular area in the brain. Measurements in post-mortem material are confounded by several factors, such as cause of death and previous medication. Fourth, the development of animal models has been hampered by the lack of comparability between the animal brain and the human brain. Not surprisingly, then, a central issue in schizophrenia research has been the difficulty in showing that a particular gene is causally related to the disease. Researchers are coming to realize that proof cannot be based on genetics alone, and any positive finding must be corroborated by additional evidence.

\section{The evidence for $A K T 1$}

Emamian et al. ${ }^{2}$ start from the general hypothesis that genetically induced alterations in the expression of protein kinases may be related to schizophrenia and should be detectable in lymphocytes. They screened for differences in protein levels of various protein kinases and phosphatases between individuals diagnosed with schizophrenia and controls. Significant differences were found for only one of the kinases studied, AKT1. They followed up this finding by comparing post-mortem brains of schizophrenic individuals with those of controls and found lower levels of AKT1 in the hippocampus and

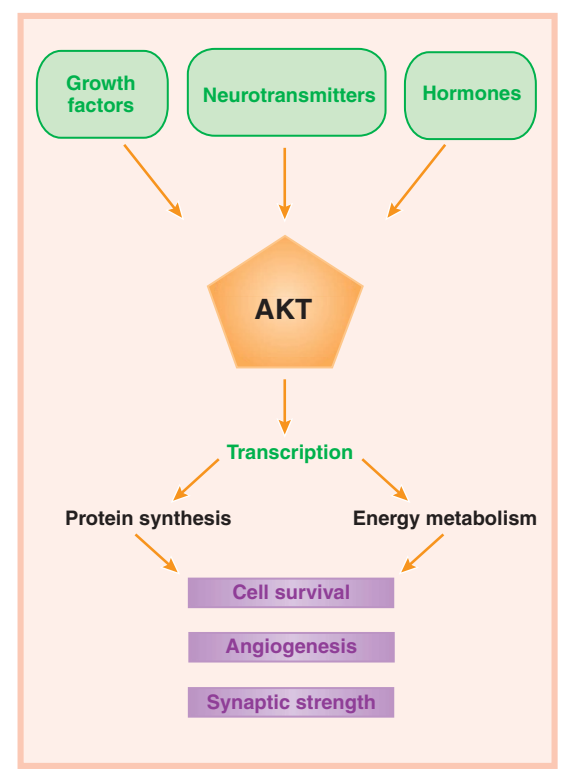

Figure 1 AKT is linked to several cellular pathways. Many different mediators interact with AKT, leading to changes in cell metabolism, cell survival and apoptosis.

frontal cortex of schizophrenic individuals. No differences were found in the two other known isoforms of AKT, AKT2 and AKT3, highlighting $A K T 1$ as a candidate gene for association with schizophrenia.

The authors next tested directly for association between sequence variants in $A K T 1$ and schizophrenia. After finding a significant positive association in a sample of 265 families with a schizophrenic proband, they went on to show that the core haplotype conferring a higher risk to schizophrenia was also associated with lower AKT1 levels in lymphocytes of controls. It is unlikely, however, that the haplotype accounts entirely for the differences in AKT1 levels between schizophrenic individuals and control samples.

But are there consequences of a decrease in AKT1 levels? To address this question, Emamian et al. ${ }^{2}$ carried out two experiments. First, they found that phosphorylation of glycogen synthase kinase- $3 \beta$ (GSK3 $\beta$ ), which is activated by AKT1, was significantly lower in schizophrenic brains. Second, they found differences in prepulse inhibition (PPI) in mice deficient for AKT1. PPI is the phenomenon in which a weak prepulse stimulus attenuates the response to a subsequent startling stimulus. Impairment of PPI has been observed in individuals with schizophrenia. The startle response can be measured easily in rodents ${ }^{3}$. A reduction of the PPI after administration of amphetamine ${ }^{2}$, which can pro- duce psychotic-like symptoms in humans, was observed in mice deficient for AKT1, but not in wild-type mice.

\section{A multifunctional protein kinase}

The door is open for speculation as to how AKT1 affects brain development and schizophrenia. AKT1 was first cloned as the cellular homolog of the $v$-act oncogene and is also known as protein kinase B. AKT1 has been extensively researched and is the subject of several thousand papers ${ }^{4}$. It has been linked to several signaling pathways mediating multiple responses (Fig. 1) and may be regulated by a large number of protein-protein interactions ${ }^{4}$.

In relation to schizophrenia, activation of AKT has been linked to the GABAergic ${ }^{5}$ and glutaminergic system ${ }^{6}$. Alterations in the GABA and glutamate systems have been described in post-mortem studies of schizophrenia for over two decades ${ }^{7}$. The two neurotransmitters are intrinsically interconnected. It has been postulated that schizophrenia may be the result of an imbalance between the inhibitory GABA and the excitatory glutaminergic neurons ${ }^{7}$. Neurons from AKT1-deficient mice are more sensitive to toxicity of kainate, a glutamate receptor agonist ${ }^{6}$. In addition, environmental stress factors (ultraviolet or hyperosmotic stress, for example) can lead to downregulation of AKT. Decreased AKT activity is related to post-ischemic neuronal damage, whereas increased activity can lead to reduced cell death ${ }^{8}$.

But GABA and glutamate are only two of many molecules suggested to be associated with schizophrenia and with AKT. No doubt the reported association between AKT1 and schizophrenia will lead to many new avenues of research. Learning more about the different roles of the three isoforms will be a crucial first step. Because of the many cellular pathways that affect AKT activity, defining the true relationship between AKT1 and schizophrenia will be challenging.

1. Bleuler, M. Dementia Praecox oder die Gruppe der Schizophrenien (Deuticke, Leipzig, 1911).

2. Emamian, E.S., Hall, D., Birnbaum, M.J., Karayiorgou, M. \& Gogos, J.A. Nat. Genet. 36, 131-137 (2004).

3. Geyer, M.A., Mcllwain, K.L. \& Paylor, R. Mol. Psychiatry 7, 1039-1053 (2002)

4. Brazil, D.P, Park, J., \& Hemmings, B.A. Cell 111, 293-303 (2002)

5. Wang, Q. et al. Neuron 38, 915-928 (2003).

6. Kim, A.H. et al. Neuron 35, 697-709 (2002).

7. Wassef, A., Baker, J. \& Kochan, L.D. J. Clin. Psychopharmacol. 23, 601-640 (2003).

8. Fukunaga, K. \& Kawano, T. J. Pharmacol. Sci. 92, 317-327 (2003). 